\title{
The trade-off between safety and flow of traffic Jingsong Meng
}

\author{
School of Computer Science and Technology, Xi'an Jiaotong University, Xi'an 100031, China \\ 462206081@qq.com
}

Keywords: automate cellular model, minimum safe distance.

\begin{abstract}
In this paper, I study how speed-limit affects the safety and flow. To study is respectively, first, I study the relationship between accidents rate and speed-limit. In this section, accidents happened due to overtaking and tailgating and the equations are based on the fundamental law of motion. Then, to study the effect of flow, I set up a model based on cellular automation. In my model, vehicles drive following series of simple rules, and the flow changes due to different speed-limit. I can draw a further conclusion by summarizing the results of my model. Finally, I can get the effect speed-limit has on flow and safety. To tradeoff the flow and safety, I allocate coefficients to each of them. The size of the coefficient represents how much I value this factor---flow or safety. Then I can understand the different results (flow and safety) based on different speed-limit.
\end{abstract}

\section{Introduction}

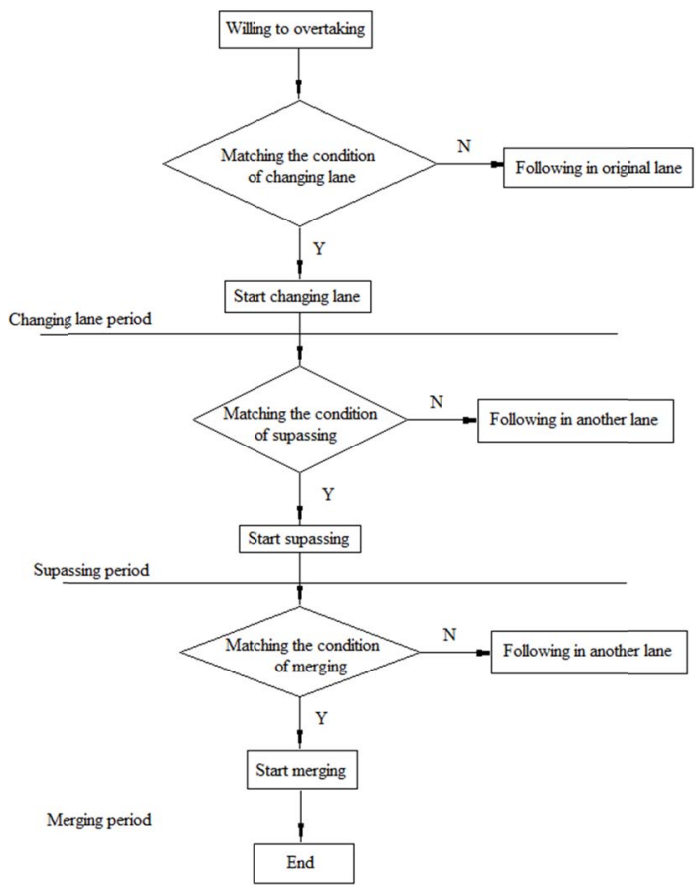

Figure 1 the flow chart of overtaking

The problems that are related to traffic flow of multi-lane highway are really complex to study. There are many scholars having conducted a lot of research. In the 1930s, J.P.Kniez firstly applied the Poisson distribution into traffic flow[1]. Then in 1936, Adams W F. considered road traffic as a random series[2]. In the early 1950s, L.A.Pipes firstly proposed traffic following model[3]. In 1953, Chandler R E, Herman R and Montroll E W used dynamic model to study traffic car following[4]. To sum up, the current research on traffic flow model is divided into four types: of the hydrodynamic model based on the continuity, the aerodynamic model based on probability and statistics, the following model based on the microscopic discrete and cellular automata model.

In this paper, at first, we have analyzed the given rule. Then, we find this rule emphasizes the single-channel driving and overtaking. So, it's necessary to analyze overtaking. The specific flow chart is show as below: 
After analyzing overtaking, we find cellular automata model is more suitable in this case. So, in this paper we build traffic flow and safety model based on cellular automata.

By the model built by us, we can find the tradeoff of traffic flow and safety easily. Meanwhile, the safety factor and traffic flow can be determined under any traffic density.

\section{Method}

As we know, in the determined traffic density, overtaking behavior has an impact on safety and traffic flow. First, we define the minimum safe following-distance and the minimum safe overtaking-distance. When the distance to vehicle in front is more than the minimum safe following-distance, the vehicle can drive in safe. When the distance to vehicle in front is more than the minimum safe overtaking-distance, the vehicle can overtake in safe. Otherwise, there is a possibility to happen accident when driving or overtaking. So, we need to calculate the minimum safe following-distance and the minimum safe overtaking-distance.

Assuming car A and car B are driving in the same lane, car A following car B. The distance between them is $\mathrm{L}$ and the speed of car $\mathrm{A}$ is $V_{A}^{0}$, the speed of car $\mathrm{B}$ is $V_{B}^{0}$. The distance of car $\mathrm{A}$ is $L_{A}$, the distance of forward car B is $L_{B}$. The maximum braking acceleration of the two vehicles is $a_{A}, a_{B}$. And $t_{d}$ is the total time when decelerating, $\mathrm{t}_{\mathrm{r}}$ is reaction time. $L_{s}$ is the safe distance between car A and car B when car A and car B has stopped.

And then, for getting the minimum safety distance of one car and other car that is been overtaken, we need to get the minimum safety distance of car following in three different conditions.

When $V_{A}<V_{B}$

$$
L_{1}=V_{A}^{0} \cdot t_{r}+\frac{\left(V_{A}^{0}\right)^{2}}{2 a_{A}}+\frac{V_{A}^{0} \cdot t_{d}-V_{B}^{0} \cdot t_{d}}{2}-\frac{\left(V_{B}^{0}\right)^{2}}{2 a_{B}}+L_{s}
$$

When $V_{A}=V_{B}$

$$
L_{2}=V_{A}^{0} \cdot t_{r}+\frac{\left(V_{A}^{0}\right)^{2}}{2}\left(\frac{1}{a_{A}}-\frac{1}{a_{B}}\right)+L_{s}
$$

When $V_{A}>V_{B}$

$$
L_{3}=V_{A}^{0} \cdot\left(t_{d}+t_{r}\right)+\frac{2 V_{A}^{0} V_{B}^{0}-2\left(V_{A}^{0}\right)^{2}-\left(V_{B}^{0}\right)^{2}}{2 a_{B}}+\frac{\left(V_{A}^{0}\right)^{2}}{2 a_{A}}-\frac{V_{B}^{0} \cdot t_{d}}{2}+L_{s}
$$

(3)

In sum, the minimum safety distance of car following is:

$$
L= \begin{cases}L_{1} & V_{A}<V_{B} \\ L_{2} & V_{A}=V_{B} \\ L_{3} & V_{A}>V_{B}\end{cases}
$$

According to the reference, the minimum safety distance of car overtaking can be calculated as formula below:

$$
d_{s}=L+L_{c i} / 2 \cos \theta-L_{c i} / 2
$$

In this formula, the $L$ represents the minimum safety distance of car following. In different condition, it equals to $L_{1}, L_{2}$ and $L_{3} . L_{c i}$ is the length of car A. $\theta$ is the angle of the car turning when overtaking.

To turn the density into the average distance between two vehicles, we can get the relationship of safe factor and traffic density. In common, the length of a cell is $7.5 \mathrm{~m}$; there is a vehicle per $7.5 \mathrm{~m}$ when density is 1 . So, the formula to calculate distance $s$ between two vehicles is shown as following: 


$$
s=\frac{7.5}{k}-L_{c i}
$$

Meanwhile, we define the ratio of the actual overtaking-distance to the minimum safe overtaking-distance as the safety factor. The formula to calculate the safety factor $h$ as following:

$$
h=\frac{s}{d_{s}}
$$

If the actual overtaking-distance is more than the minimum safe overtaking-distance, we regard the safety factor is 1 , otherwise, the safety factor varies from 0 to 1 . Then, we need to study the changes in traffic flow in different overtaking-distance. The larger safety factor represents the condition more safe.

In this section, we use the theory of cellular automata to analyze the relationship between traffic flow and traffic density.

Automate cellular model is a model of traffic flow that time and space are discrete. Vehicles are randomly distributed on one-dimensional discrete cellular chain of the length $\mathrm{L}$, and each cell can only be occupied by a vehicle at any time. $v_{i}, x_{i}$ and $d_{i}$ represent the speed, the position of cellular and the vehicle spacing of $i$ car at $t$ time. And $v_{i}=v_{\min }, v_{\min }+1, \ldots, v_{\max } d_{i}=x_{i+1}-x_{i}+1$.

In the driving process, we have developed the following rules:

In the condition that $v_{i} \leq v_{\max }$ and the distance between $i$ car and the formal car is less than the safe distance $L_{2}$ (calculated by formula (2)), the $i$ car will do uniformly accelerated motion at the acceleration having been set. $v_{i} \rightarrow v_{\max }$

When having reached the speed high limit, the $i$ car will keep driving at $v_{\max } \cdot x_{i} \rightarrow x_{i}+v_{i}$.

When the distance from the vehicle in front less than a given safe distance, if the vehicle in front speeds large, doing uniform deceleration to a safe distance. $v_{i} \rightarrow \min \left(v_{i}, d_{i}\right)$.

These rules control the forward movement of the vehicle, in a multi-lane traffic we also need to introduce lane-changing rules, which controls the lane changing behavior of the vehicle in the driveway.

Considering the rule given, cars are just able to drive in one lane, and to overtake other car in the other lane. After overtaking, cars need to return the original lane. So, we have developed some lane-changing rules:

When the distance from the vehicle in front more than the given distance for overtaking, the car will overtake the vehicle in front.

For those vehicles driving in overtaking lane, they should keep checking whether there is enough space to return in original. If so, then to return; if not, to accelerated at the acceleration having been set, until finding a suitable location to return.

Under the premise of following these rules, we set on a series of time interval, in which there is a car starting in the beginning of original lane. These time intervals represent traffic density. At the end of original lane, to calculate the number of vehicles in 180- seconds time interval, and to regard this number as traffic flow. Then, we can get the relationship of traffic flow and the overtaking-distance in different traffic density.

\section{Results}

First, we select $60 \mathrm{~km} / \mathrm{h}$ as $v_{\min }$ of overtaking-lane and original lane, $100 \mathrm{~km} / \mathrm{h}$ as $v_{\max }$ of original lane and $120 \mathrm{~km} / \mathrm{h}$ as $v_{\max }$ of original lane. The initial speeds of vehicles range from $v_{\min }$ to $v_{\max }$ that are randomly selected.

Then, to consider safety factor, we need to calculate the minimum safe following-distance and the minimum safe overtaking-distance by speed of adjacent vehicle. Due to having provided car driving rules, we can think that the minimum safe following-distance should be calculated when $V_{A}=V_{B}$. So, we calculate the minimum safe following-distance only by the speed of this car. 
According to the related literature issued by Ministry of Transportation Highway Administration, the reaction time and the brake initial time $t_{r}$ is normally $0.9 \mathrm{~s}$, the total time when decelerating $t_{d}$ is normally $0.15 \mathrm{~s}$. The maximum braking acceleration of the two vehicles $a_{A}, a_{B}$ is range from 6.86 to $7.84 \mathrm{~m} / \mathrm{s}^{2}$. In this paper, the value of $a_{A}$ is $7.84 \mathrm{~m} / \mathrm{s}^{2}$, the value of $a_{\mathrm{B}}$ is $6.86 \mathrm{~m} / \mathrm{s}^{2} . L_{s}$ is the safe distance between car A and car B when car A and car B has stopped. According to the provisions of the 'Road Users Code' in China, $L_{s}=0.5 \mathrm{~m}$. Meanwhile, generally speaking, the mean of $L_{c i}$ is $4.2 \mathrm{~m}$, $\theta$ is $10^{\circ}$.

Calculated by $\mathrm{C}++$, we can get the curve of speed and traffic density as following figure:

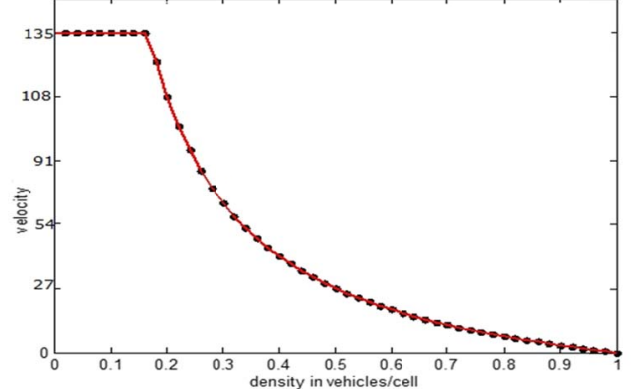

Figure 5 the curve of speed and traffic density

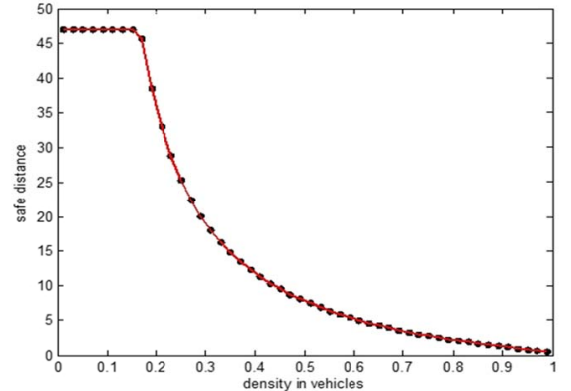

Figure 6 the curve of $d_{s}$ and traffic density

Figure 5: In this figure, 1 to 5 namely represent $24 \mathrm{~km} / \mathrm{h}, 48 \mathrm{~km} / \mathrm{h}, 72 \mathrm{~km} / \mathrm{h}, 96 \mathrm{~km} / \mathrm{h}, 120 \mathrm{~km} / \mathrm{h}$. From this figure, we can see when traffic density is not so large, the speed keep constant, and when traffic density keep increasing, the speed begin to decrease to zero. In this case, the speed is $135 \mathrm{~km} / \mathrm{h}$. The density which turning point in the figure corresponds to is the free flow density, 0.16 vehicle/cells.

According to formula (2) and formula (5), we can get the minimum safe overtaking-distance as following:

$$
d_{s}=\frac{\left(V_{A}^{0}\right)^{2}}{2}\left(\frac{1}{a_{A}}-\frac{1}{a_{B}}\right)+L_{s}+L_{c i} / 2 \cos \theta-L_{c i} / 2+V_{A}^{0} \cdot t_{r}
$$

So, we can get the curve of $d_{s}$ and traffic density as following figure:

Figure 6: The curve of $d_{s}$ and traffic density is similar with the curve of speed and traffic density. When traffic density is not so large, the $d_{s}$ keep constant, and when density traffic keeps increasing, $d_{s}$ begins to decrease to a constant.

To turn the density into the average distance between two vehicles referring to formula (6) and formula (7), we can get the curve of safe factor and traffic density as following:

Figure 7: In this figure, we can see that: when the density is small, safety factor keep constant; when the density keep increasing, safety factor decrease firstly and then increase. It shows when density is not so large, overtaking is always safe; with the density increasing, at the same time, the speed being not so small, the possibility of happening accident is increasing; when the density consisting on increasing, due to speed decreasing quickly, the safety factor increase. The trend of this curve is realistic.

At the same time, we can get the curve of traffic flow and traffic density:

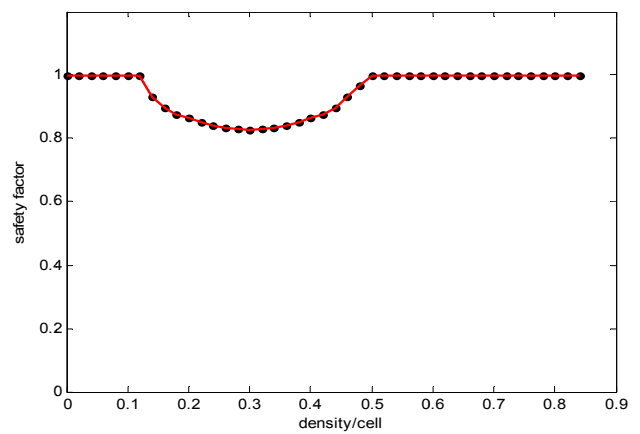

Figure 7 the curve of safe factor and traffic density

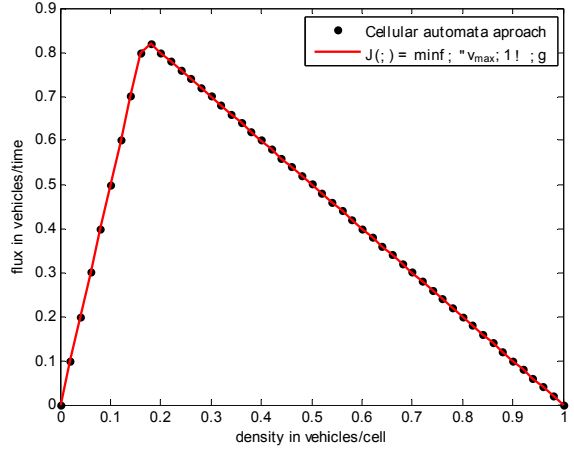

Figure 8.the curve of traffic flow and traffic density 
Figure 8: From this figure, we can see with the increase of traffic density, the traffic flow increase first and decline next. When traffic density is 0.18 cells, flow reach the maximum, 0.82 .

Then, comprehensively study traffic flow and safety to find the tradeoff of them.

As we all know, safety is really important. Thus, we think when finding the tradeoff, the proportion of safety is more than traffic flow. Thus, we define the proportion of safety is 0.7 and the proportion of traffic flow is 0.3 . Then, we can get the curve of comprehensive factor and traffic density:

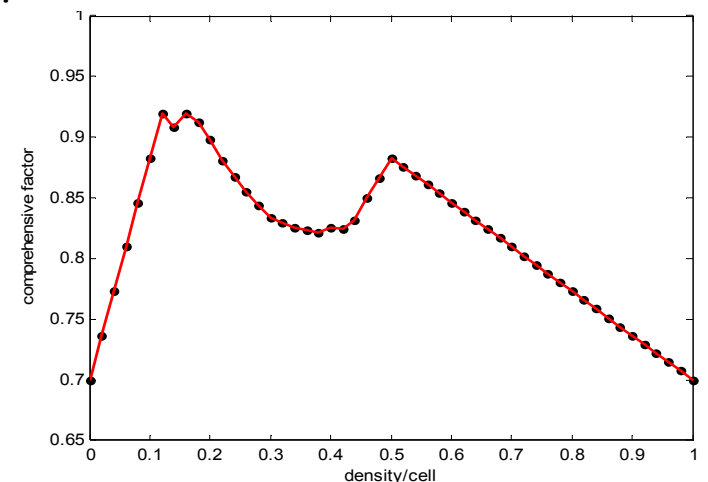

Figure 9 the curve of comprehensive factor and traffic density

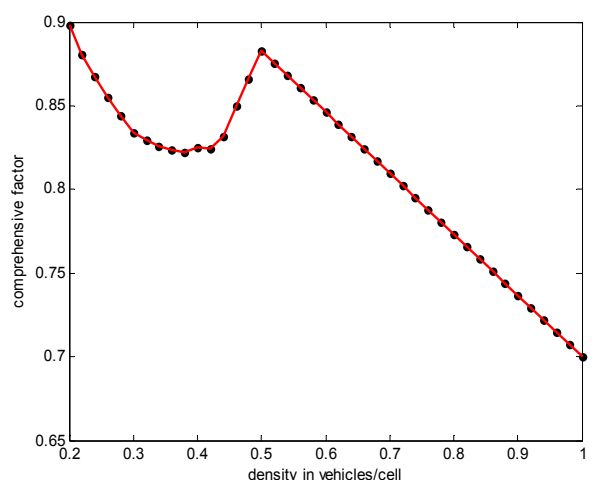

Figure 10 the curve when the high speed limit is $120 \mathrm{~km} / \mathrm{h}$

Figure 9: In this figure, we can see that the comprehensive factor firstly increases with the density increasing, then declining a litter and then increasing. When density reached 0.5 cells, the density keep declining. This moment, we do not take into account the speed limit. That is to say, the speed is range from 0 to $135 \mathrm{~km} / \mathrm{h}$.

Actually, we need to consider the speed limit. When the high speed limit is $120 \mathrm{~km} / \mathrm{h}$, the density is range from 0.2 cells to 1 cell; when the high speed limit is $80 \mathrm{~km} / \mathrm{h}$, the density is range from 0.26 cells to 1 cell. The curves under different high speed limit as following:

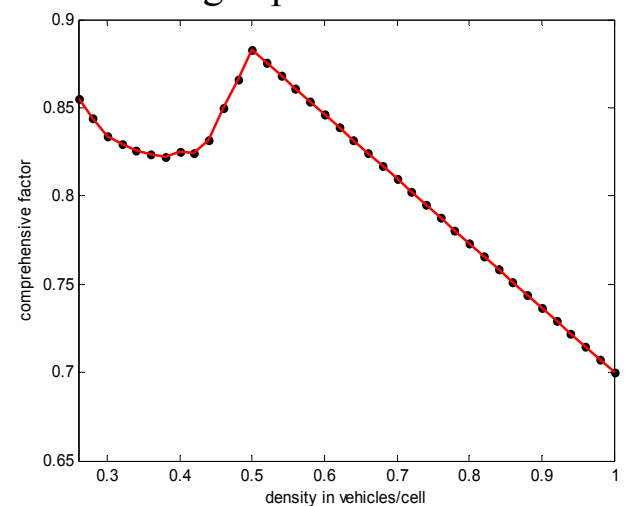

Figure 11. The curve when the high speed limit is $80 \mathrm{~km} / \mathrm{h}$

\section{Conclusion}

So, we can preliminarily reach these conclusions: When the high speed limit is $120 \mathrm{~km} / \mathrm{h}$ and the density is less than 0.2 cells, the given rule performance good. When high speed limit is $80 \mathrm{~km} / \mathrm{h}$ and the density is less than 0.5 cells, the given rule performs well. Then, we need to find other rules for better traffic flow and safety, as well as to correct our conclusion.

\section{References}

[1] Kinzer J P. Application of the theory of probability to problem of highway traffic [Dissertation]. Politech. Inst. Brooklyn: B.C.E, 1933.

[2] Adams W F. Road traffic considered as a random series. J.Inst. Civil Eng. 1936, 4: 121-130. 
[3] Pipes L A. An operational analysis of traffic dynamics. J. Appl. Phys, 1953, 24:274-287

[4] Chandler R E, Herman R, Montroll E W, Traffic dynamics: studies in car following. Oper. Res, 1958, 6:165-184. 